\title{
Activity and Stability Studies of Verbascoside, a Novel Antioxidant, in Dermo-Cosmetic and Pharmaceutical Topical Formulations
}

Silvia Vertuani ${ }^{1,2, *}$, Erika Beghelli ${ }^{1}$, Emanuela Scalambra ${ }^{1}$, Gemma Malisardi ${ }^{1}$, Stefano Copetti ${ }^{3}$, Roberto Dal Toso ${ }^{3}$, Anna Baldisserotto ${ }^{1}$ and Stefano Manfredini ${ }^{1,2}$

1 Department of Pharmaceutical Sciences, University of Ferrara, Via Fossato di Mortara 17-19, 44121 Ferrara, Italy

2 Ambrosialab, University of Ferrara, Via Mortara 171, 44121 Ferrara, Italy

3 Biotechnological Research Institute (I.R.B.), Via Lago di Tovel, 7, 36077 Altavilla Vicentina (VI), Italy

* Author to whom correspondence should be addressed; E-Mail: vrs@unife.it; Tel.: +39-0532-455294.

Received: 27 June 2011; in revised form: 10 August 2011 / Accepted: 11 August 2011 /

Published: 18 August 2011

Abstract: We here report the results of our investigations carried out on verbascoside, a phenylpropanoid glycoside known for its antioxidant, anti-inflammatory and photoprotective actions. Verbascoside was obtained from Buddleia davidii meristematic cells, obtained in turn using a sustainable biotechnology platform which employs an in vitro plant cell culture technology. Verbascoside was first investigated to assess the behaviour of the active ingredient in solution or in finished preparations, in view of its potential topical use, especially in skin protection. Stability studies were performed by HPLC, and a PCL assay was adopted to determine the radical scavenging activity toward superoxide anion. The high hydrophilic character of verbascoside, suggested in a somewhat limited range of possible applications, leading us to explore its derivatization to obtain the semi-synthetic derivative VPP, an acyl derivative of verbascoside, with an improved range of applications due to its lower hydrophilic profile. Alone, VPP revealed increased antioxidant activity, both as an active ingredient and in dermocosmetic preparations. Stability studies showed a greater stability of VPP in lipophilic vehicles, whereas the parent verbascoside proved more stable in an $\mathrm{O} / \mathrm{W}$ emulsions. Verbascoside was also stable in suppositories, an interesting pharmaceutical form for possible applications in treatment of inflammation of the intestinal mucosa. 
Keywords: verbascoside; VPP; antioxidant activity; PCL; HPLC analysis; stability in dermocosmetic formulations; suppositories; sustainability; meristematic cells

\section{Introduction}

Oxidative stress is considered to play an essential role in the pathogenesis of aging and degenerative diseases [1,2]. It results in oxidative alteration of biological macromolecules such as lipids, proteins and nucleic acids. In order to cope with an excess of free radicals produced upon oxidative stress, human body has developed sophisticated mechanisms for maintaining redox homeostasis. These protective mechanisms include scavenging or detoxification of reactive oxygen species (ROS), blocking ROS production, sequestration of transition metals, as well as enzymatic and non-enzymatic antioxidant defences that are endogenous [3] when produced in the body or exogenous if supplied with the diet. The skin, a highly metabolic tissue that possesses the largest surface area in the body, represents the major target for free radical damage. Particularly, it is well known that free radicals and ROS are involved in the mechanisms leading to cutaneous damages, such as early ageing, inflammatory disorders and skin cancers. Over the last decade antioxidants have been proposed as functional ingredients for anti-aging preparations, and to prevent and modulate oxidative skin damage. Market demands, increasingly oriented towards natural antioxidants, have also fostered the research and development of innovative cosmeceutical ingredients of vegetable origin with radical-scavenger activity. In this context and prompted by our continuous interest in this field, we have recently investigated extracts of butterfly bush (Buddleja davidii), a shrub in the Buddlejaceae family widely used as an ornamental plant and known in traditional medicine for their wound healing, anti-inflammatory, diuretic, anti-allergic detergent, antiviral and antibacterial properties. The Buddleja davidii extract used in the present investigation derives from a biotechnology platform, which employs in vitro plant cell culture technology [4]. This biotechnology method allows one to obtain plant extracts enriched in the secondary metabolites, at concentrations that cannot be obtained by traditional methods (i.e., extraction from wild plants, synthesis). The principal components of the Buddleia davidii extracts, were identified as phenylpropanoid derivatives such as verbascoside, isoverbascoside, leucosceptoside A and martynoside (Figure 1), together with a further component constituted mainly by oligo- and polysaccharides, proteins and lipids (chromophore-free fraction).

Figure 1. Chemical structure of Buddleia davidii phenylpropanoids: verbascoside, isoverbascoside, martynoside and leucosceptoside A. Cinnamic and phenylethanolic moieties are highlighted by red and blue colour respectively.

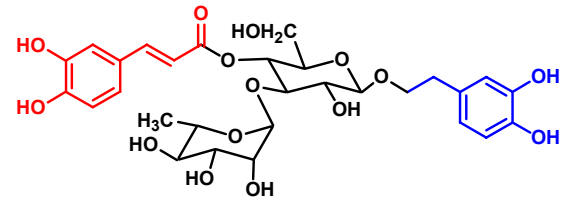

Verbascoside

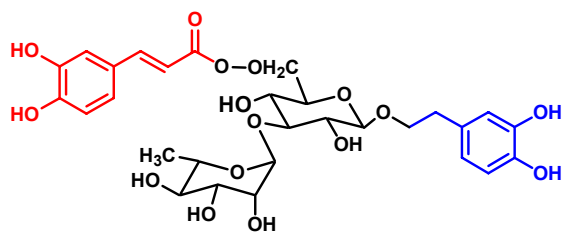

Isoverbascoside

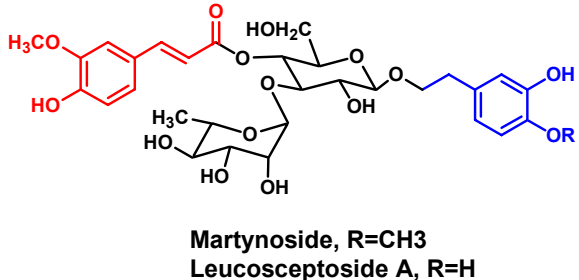


Verbascoside is the most abundant of these chemical substances and may be found in very high concentrations (approximately 80\%) in Buddleja davidii cell cultures. The biological properties of verbascoside, also known as acteoside, have been described in the literature and comprise a wide spectrum of activities, including antioxidant, anti-inflammatory, photoprotective and chelating actions.

In particular, a significant antioxidant effect of verbascoside has been recently documented by Aleo et al. in an experimental study comparing numerous natural antioxidant substances using various method of determination [5-8]. Furthermore the anti-inflammatory activity of verbascoside has been confirmed by an in vitro test performed on cell cultures of primary human keratinocytes [7], in which verbascoside was able to significantly reduce, in a dose-dependent manner, the release of pro-inflammatory chemokines. This study has also demonstrated that verbascoside promotes skin repair and ameliorates skin inflammation due to its ROS scavenging, antioxidant, iron chelating, and glutathione transferase (GST) activity inducing properties $[9,10]$. An in vivo study, conducted on inflammation of the intestinal mucosa, demonstrated that verbascoside is able to inhibit the activation of pro-inflammatory proteins and consequently the enzymatic activity of matrix metalloproteinases, the latter also being involved in skin aging phenomena. The results of this study suggested that verbascoside functions as an intracellular radical scavenger and thus reduces the microscopic and macroscopic signs of colitis in the rat. Therefore, administration of verbascoside may be beneficial for the treatment of inflammatory bowel disease [8].

Recently, results have clearly indicated that the anti-inflammatory activity of verbascoside in a dinitrobenzenesulfonic acid (DNBS)-induced inflammatory bowel disease model can be enhanced by peroxisome proliferator-activated receptor (PPAR)- $\alpha$. These observations suggest that verbascoside could use the same pathway as PPAR- $\alpha$ agonists in inflammatory diseases [11].

Based on these interesting data, and in continuation of our efforts toward studying this interesting molecule [12], we started the current investigation with the objective of verifying the behavior of the active ingredient in solution or in dermocosmetic preparations, in view of its potential use in cosmetics. Stability studies were performed by HPLC and took into consideration solutions at different $\mathrm{pH}$ value and storage conditions or cosmetic formulations containing the active. The antioxidant activity was determined by a PCL assay. Despite the good antioxidant activity measured, the highly hydrophilic character of the active ingredient was envisaged by us as a limit in its application in different types of formulations. In order to overcome this limitation, we decided to attempt simple chemical modifications of the molecule in order to make it more lipid-soluble and thus incorporable into the lipophilic phase of the cosmetic formulations, which represents a more stable matrix. This approach, usually undertaken to increase the stabilization of water-soluble ingredients, has been successfully applied to unstable products such are ascorbic acid, tocopherol and retinol [13]. Stabilization is generally obtained by esterification, so that the newly synthesized lipophilic molecules may be converted chemically or enzymatically in their bioactive form in the site of application.

In this paper, we report the results of this experiment and the stability-activity studies carried out on VPP, obtained by semi-synthetic approach, in the effort to reduce the hydrophilic profile of parent molecule without affecting the required antioxidant activity. Finally, verbascoside itself was also enclosed in a pharmaceutical topical lipid form (suppositories), and the stability analysis was conducted with the aim to determine suitability for a possible use in the treatment of inflammation of 
intestinal mucosa. A preliminary investigation conducted on VPP in the same formulation showed promising results.

\section{Results and Discussion}

Phenylpropanoid compounds are characterized by cinnamic and phenylethanolic moieties linked to the same glucopyranose molecule (usually glucose) by an ester and glycosidic bond, respectively [16]. The bioactive structure of these molecules which is responsible for the antioxidant [17,18], antimicrobial $[19,20]$, anti-inflammatory $[7,9,10]$ and antimutagenic activities $[21]$ is thought to be the aglycone. Verbascoside, by virtue of its chemical structure, is highly soluble in hydrophilic media. However, due to the presence in solution of radical forms of oxygen or nitrogen, its hydroxyl groups easily participate in oxidation processes that in turn lead to new species with loss of the UV absorption at a wavelength of $330 \mathrm{~nm}$ characteristic of catechol, hydroxytyrosol and caffeic acid. Taking all this into consideration, we have decided to modify verbascoside's structure in order to obtain a more lipophilic molecule, with the aim of increasing the range of applications, and then to evaluate its stability and anti-oxidant properties in some topical formulations. Thus, all the hydroxyl moieties in verbascoside were esterified using an acyl chloride in the presence of 4-dimethylaminopyridine (4-DMAP) and subsequently selectively deprotected to phenolic hydroxyls, using $N, N, N$-triethylamine (TEA) in methanol to afford VPP (Scheme 1).

Scheme 1. Synthesis of the pentasubstituted derivative of verbascoside (VPP).

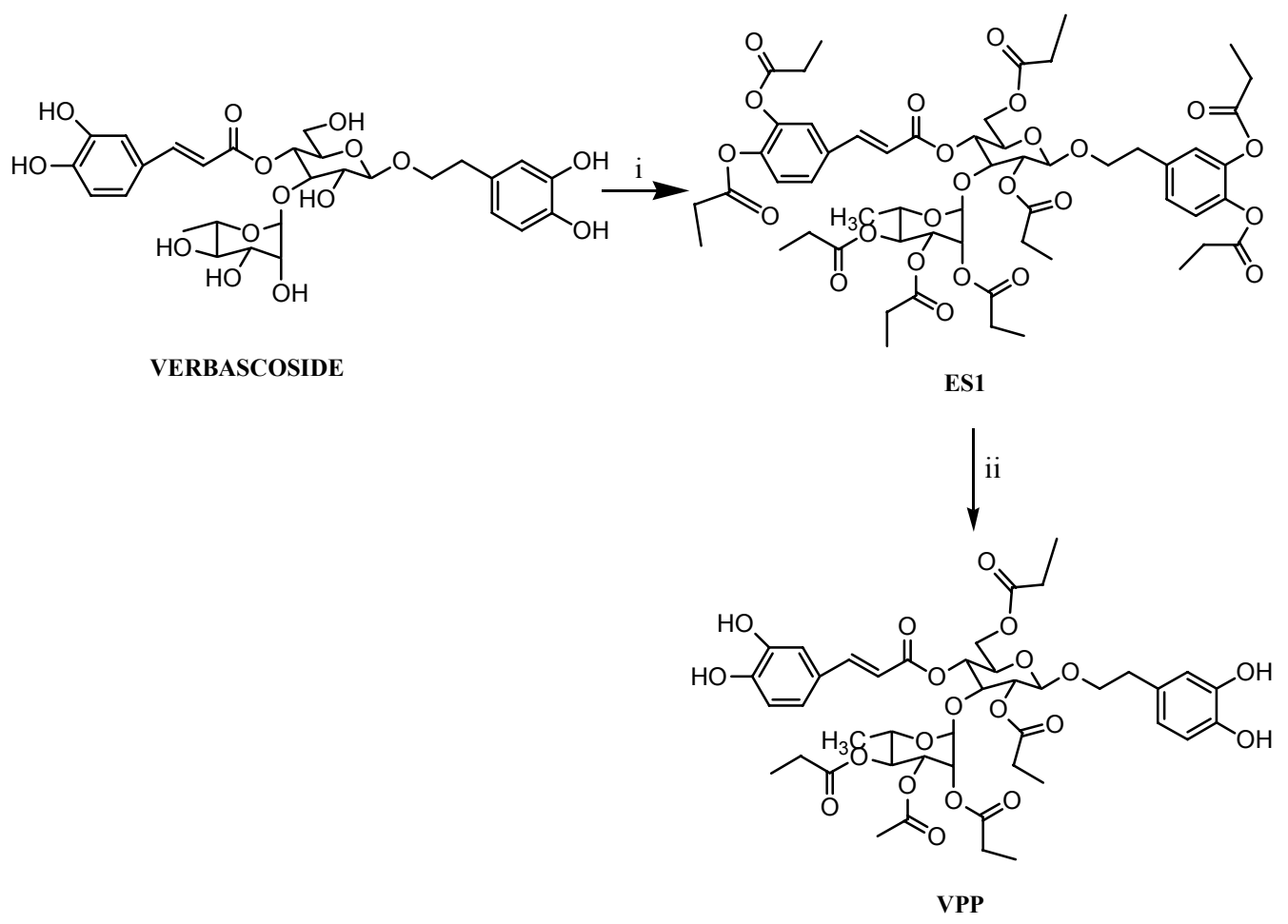

Reagents: i: DMAP, propionyl chloride, $\mathrm{CH}_{2} \mathrm{Cl}_{2}$, ii: TEA, $\mathrm{MeOH}$.

We first conducted a stability study with the aim of evaluating the behaviour of verbascoside and VPP as a function of time, $\mathrm{pH}$ and of the storage conditions. Verbascoside was analyzed in $\mathrm{EtOH}-\mathrm{H}_{2} \mathrm{O}$ solution $(80: 20, \mathrm{v} / \mathrm{v})$ and in buffer solutions at $\mathrm{pH} \mathrm{7,6}$ and 5. All the solutions were checked at room 
temperature kept in the dark and in oven subjected to accelerated aging at $40{ }^{\circ} \mathrm{C}$. The results show that verbascoside in buffered solution at $\mathrm{pH} 7$, after just three weeks of preservation at room temperature and two weeks in oven was completely degraded, while in the solution at $\mathrm{pH} 6$ its degradation is complete in 60 days.

The sample at $\mathrm{pH} 5 \mathrm{kept}$ in the dark showed the best verbascoside recovery percentages over time (73\%), confirming its greater stability in a weak acid medium. Verbascoside also presents quite good stability in EtOH- $\mathrm{H}_{2} \mathrm{O}$ solution (80:20, v/v) at room temperature (Figure 2).

Figure 2. Verbascoside stability in solution at different $\mathrm{pH}$ value and storage conditions.

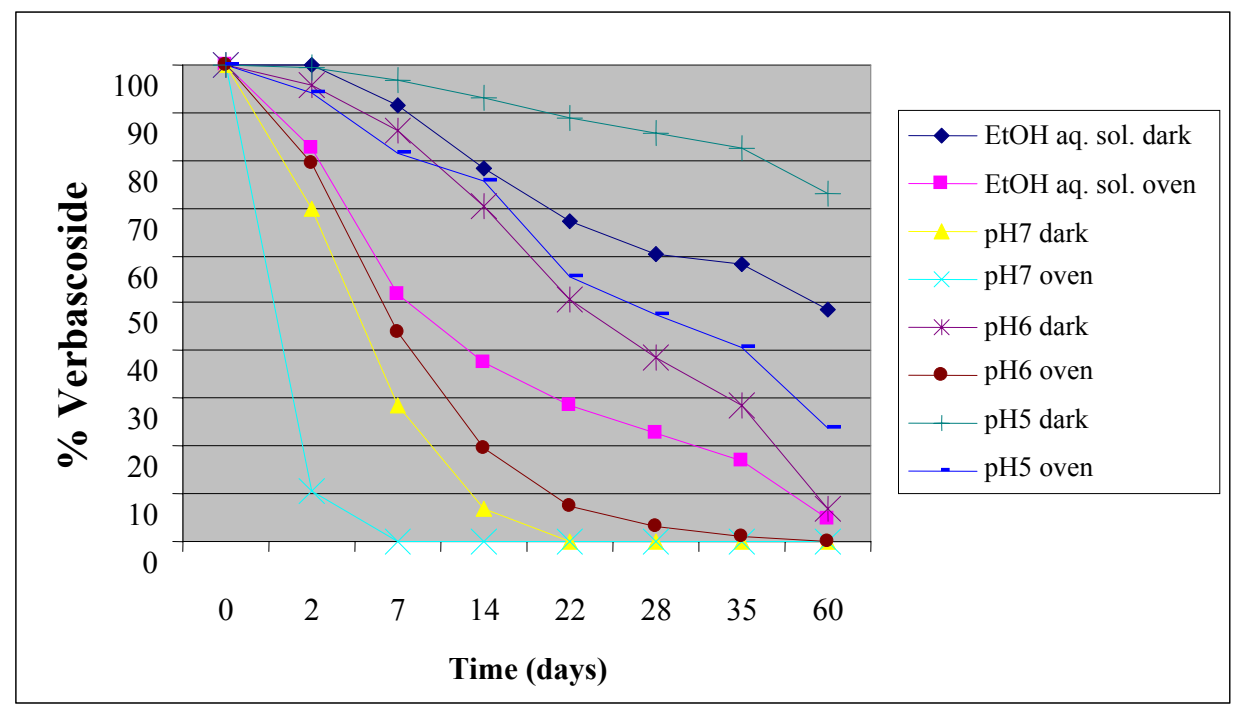

Regarding VPP, we have estimated its behaviour in $\mathrm{EtOH}-\mathrm{H}_{2} \mathrm{O}$ solution $(80: 20 \mathrm{v} / \mathrm{v})$ because it was not soluble in the buffer solutions used for the verbascoside study. The results show that after just one week subjected to accelerated aging, VPP is completely degraded, while at room temperature and kept in the dark its degradation is complete in 60 days (data not shown).

In order to measure the lipophilic properties of the molecules, their partition coefficient $(\operatorname{LogP})$, calculated as the logarithm of the ratio of concentrations of the substance in octanol and water, was evaluated. This determination was conducted in a biphasic system of octanol $(20 \mathrm{~mL})$ and water $(20 \mathrm{~mL}$ to $\mathrm{pH} 6)$ at $25{ }^{\circ} \mathrm{C}$. After 30 minutes of stirring, the biphasic mixture was centrifuged for 10 minutes at 4,000 rpm to complete separation of phases and then $5 \mathrm{~mL}$ of each phase was used for spectrophotometric determination (at $330 \mathrm{~nm}$ ) of the concentrations of verbascoside $(5 \mathrm{mg} / 20 \mathrm{~mL})$ at equilibrium. A similar analysis has been done for VPP $(5 \mathrm{mg} / 20 \mathrm{~mL})$. The data are described in Table 1 and show a significant difference in the distribution of VPP, higher in octanol than in water, (0.96 $\log \mathrm{P}$ ), wherease verbascoside was less concentrated in octanol than water with a $\log \mathrm{P}-0.56$. These data suggest that VPP, being more lipophilic than verbascoside, accumulates in the lipid phase of the formulations thus reversing both the solubility and active formula compartment. 
Table 1. Partition coefficients of verbascoside and VPP in a biphasic mixture of octanol and water.

\begin{tabular}{|c|c|c|c|}
\hline & Octanol $(\mathrm{mg} / 20 \mathrm{~mL})$ & $\mathrm{H}_{2} \mathrm{O}(\mathrm{mg} / 20 \mathrm{~mL})$ & $\log P *$ \\
\hline Verbascoside & 1.07 & 3.93 & -0.56 \\
\hline VPP & 4.50 & 0.50 & 0.96 \\
\hline
\end{tabular}

$* \log \mathrm{P}=\log 10[\mathrm{mg} / 20 \mathrm{~mL}]$ octanol $/[\mathrm{mg} / 20 \mathrm{~mL}] \mathrm{H}_{2} \mathrm{O}$.

The two compounds were then tested to determine their antioxidant capacity by PCL analysis. Surprisingly, in the case of the semi-synthetic derivative VPP, higher antioxidant activity was observed as compared to that of verbascoside (13.38 and $10.12 \mathrm{mmol}$ Trolox/mmol respectively) (Figure 3).

Figure 3. PCL analysis of verbascoside and VPP. Each value was obtained from three experiments (mean $\pm \mathrm{SE})$.

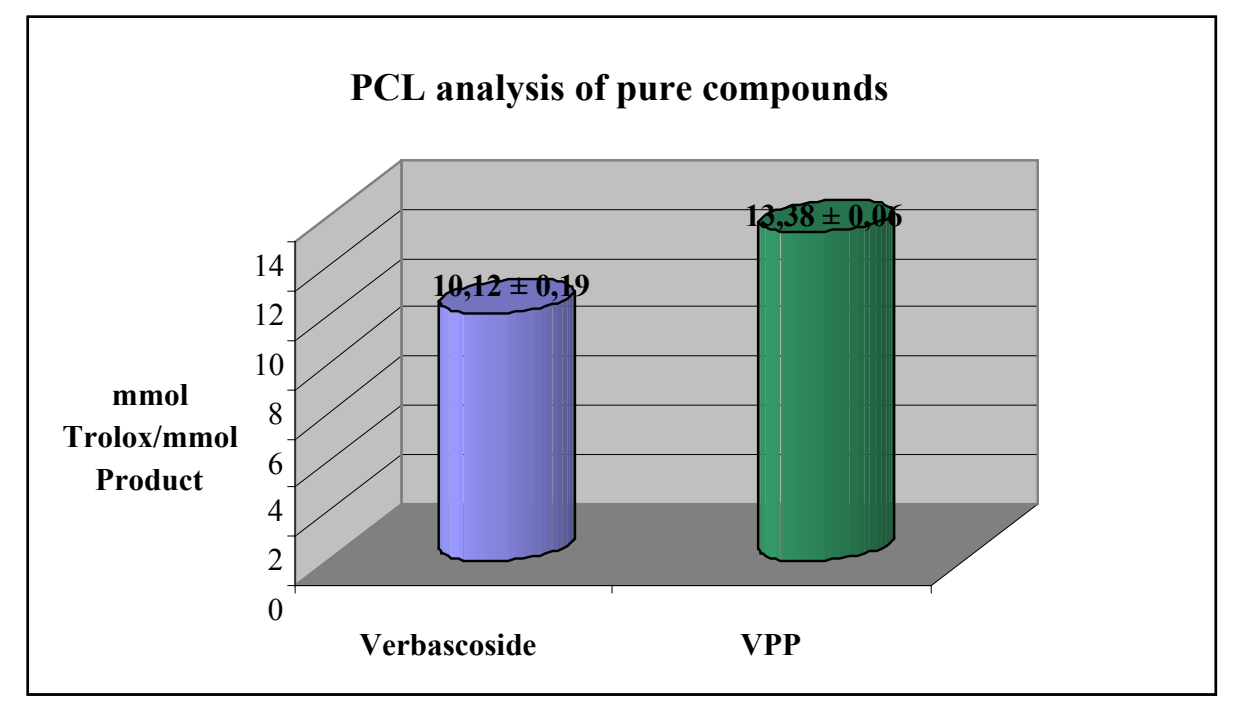

Starting from this interesting data we have decided to continue the study by the investigation of the antioxidant activity and the stability of the active components incorporated into finished dermocosmetic formulations at $0.3 \%(\mathrm{p} / \mathrm{p})$ and subjected to accelerated aging in oven at $40{ }^{\circ} \mathrm{C}$.

Two different cosmetic formulations, Formulation 1 (a cosmetic base made with emollients, emulsifiers, and other ingredients that do not have any antioxidant properties) and Formulation 2 (made with water free ingredients) were employed. PCL analysis of the two cosmetic bases have confirmed the higher anti-oxidant activity of the formulation containing VPP than verbascoside (14.63 and 13.7 nanomol Trolox/mg formulation respectively) (Figure 4). PCL analysis of the same formulations over 150 days in oven have revealed a maintenance of the anti-oxidant activity (data not shown).

To complete the study, formulations 1 and 2 containing the active ingredients, were submitted to accelerated aging in oven at $40{ }^{\circ} \mathrm{C}$ and monitored by HPLC to evaluate the stability of verbascoside and VPP in function of time. The investigation highlighted that the concentration of verbascoside decreases by about 30\% in both the formulations 1 and 2 (Figure 5) over 150 days in the oven. 
Figure 4. PCL analysis of formulations 1 with verbascoside and VPP. Each value was obtained from three experiments (mean $\pm \mathrm{SE}$ ).

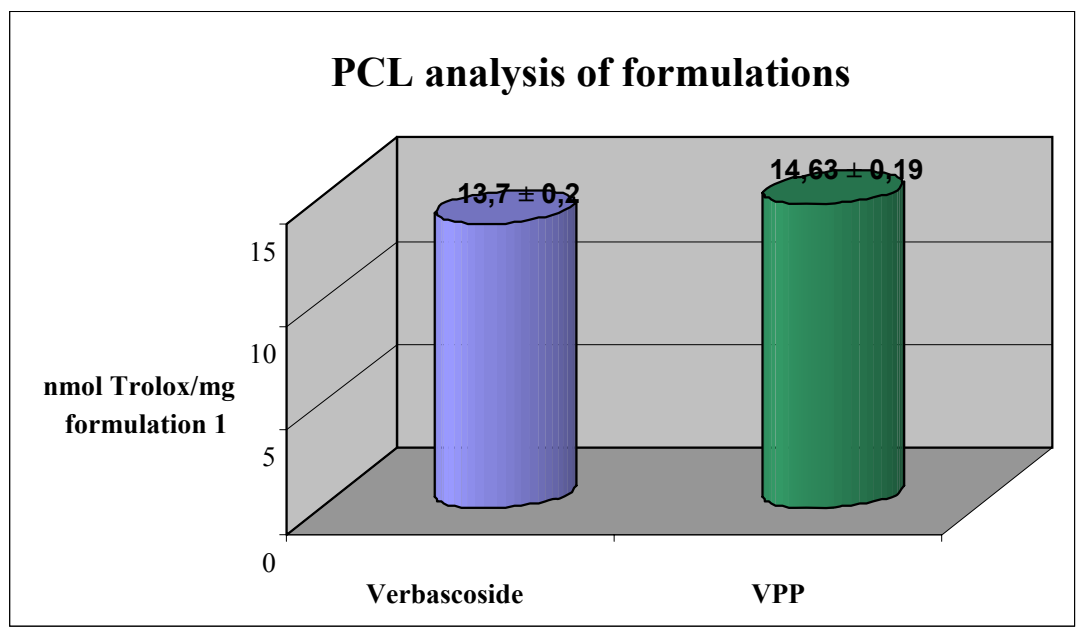

Figure 5. Verbascoside stability in the different formulations stored at $40{ }^{\circ} \mathrm{C}$. Each value was obtained from three experiments (mean $\pm \mathrm{SE}$ ).

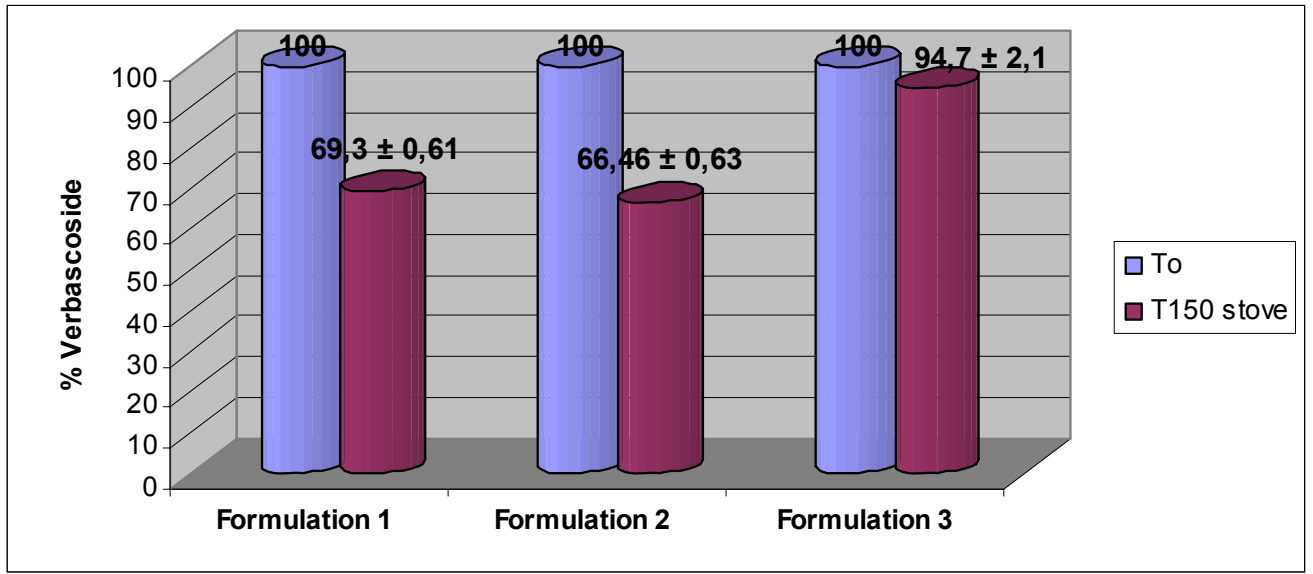

Figure 6. VPP recovery rate in the different formulations stored at $40{ }^{\circ} \mathrm{C}$. Each value was obtained from three experiments (mean $\pm \mathrm{SE}$ ).

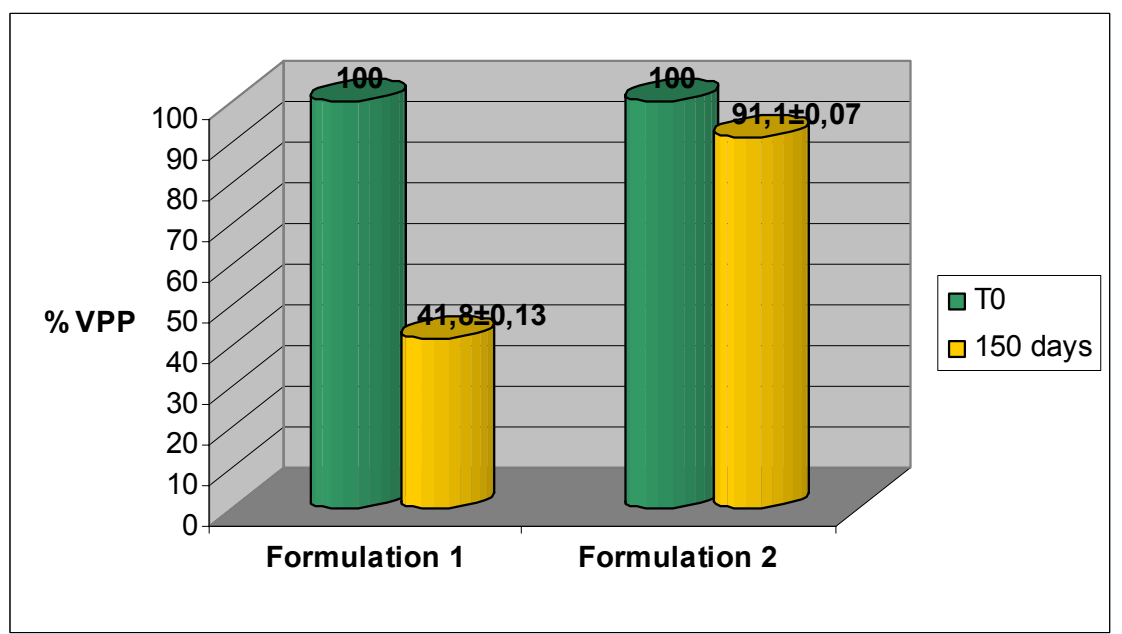


Regarding VPP, we have observed over 150 days in oven a decrease of about $60 \%$ in the formulation 1, while in the formulation 2 the active ingredient is significantly stable. In fact the decrease of VPP in this formulation is less than 10\% (Figure 6).

It is well known that verbascoside has a wide spectrum of activities, including anti-inflammatory actions, so we have finally decided to incorporate the active substance in a third formulation like suppositories $(0.3 \% \mathrm{p} / \mathrm{p})$ with the aim to investigate its stability in a pharmaceutical lipidic form for possible future applications to the inflammation of the intestinal mucosa. We observed that after 150 days in oven at $40{ }^{\circ} \mathrm{C}$ the decrease of verbascoside was only $6 \%$ (Figure 5 ). A preliminary study of stability in this kind of formulation was conducted also for VPP: data show that after 30 days in oven VPP is $100 \%$ stable (data not shown).

\section{Experimental}

\subsection{Materials and Methods}

\subsubsection{General}

All reagents were from Sigma-Aldrch srl (Milan, Italy). Reaction course was routinely monitored by thin-layer chromatography on precoated silica gel plates (Macherey-Nagel Durasil-25) by detection under a 254-nm UV lamp and/or by spraying the plates with $\mathrm{FeCl}_{3}$ solution or dilute potassium permanganate solution. Column chromatography was performed with Macherey-Nagel 0.063-0.2 mm/70-230 mesh silica gel. The molecular weights of the compounds were determined by ESI (Micromass ZMD 2000), and the values are expressed as $[\mathrm{MH}]^{+} .{ }^{1} \mathrm{H}-\mathrm{NMR}$ spectra were obtained using a Mercury Plus400 spectrometer. HPLC analysis was performed using an Agilent 1100 Series HPLC System equipped with a G1315A DAD and with an Hydro RP18 Sinergi 80A column $(4.6 \times 150 \mathrm{~mm}, 4 \mu \mathrm{m})$ from Phenomenex.

\subsubsection{Synthesis of Verbascoside Nonaproprionate (ES1)}

To a pre-cooled $\left(0{ }^{\circ} \mathrm{C}\right)$ solution of verbascoside $(400 \mathrm{mg}, 0.64 \mathrm{mmol})$ and DMAP $(2.81 \mathrm{~g}$, $23.04 \mathrm{mmol})$ in $\mathrm{CH}_{2} \mathrm{Cl}_{2}(40 \mathrm{~mL})$, propionyl chloride $(17.28 \mathrm{mmol})$ was slowly added. After stirring at room temperature for $14 \mathrm{~h}$ the reaction mixture was washed with $\mathrm{H}_{2} \mathrm{O}$, saturated $\mathrm{NaHCO}_{3}$ and with Brine. The organic phase was then dried $\left(\mathrm{Na}_{2} \mathrm{SO}_{4}\right)$, filtered and the solvent evaporated under reduced pressure. Purification of the crude residue over silica gel column chromatography (eluent: $\mathrm{CH}_{2} \mathrm{Cl}_{2} / \mathrm{MeOH}, 99 / 1, \mathrm{v} / \mathrm{v}$ ), afforded ES1 (72\% yield) as a white foam. ${ }^{1} \mathrm{H}-\mathrm{NMR}$ (400 $\mathrm{MHz}$, DMSO- $\left.d_{6}\right): \delta(\mathrm{ppm}) 0.79\left(\mathrm{t}, 3 \mathrm{H}, \mathrm{CH}_{3}\right) ; 0.90-1.03\left(\mathrm{~m}, 15 \mathrm{H}, 5 \mathrm{x} \mathrm{CH}_{3}\right) ; 1.09-1.15\left(\mathrm{~m}, 12 \mathrm{H}, 4 \mathrm{x} \mathrm{CH}_{3}\right)$; 1.95-2.05, 2.16-2.25, 2.3-2.39 (m, 10H, 5x CH$) ; 2.55-2.60\left(8 \mathrm{H}, 4 \mathrm{x} \mathrm{CH}_{2}\right) ; 2.75-2.85(\mathrm{~m}, 2 \mathrm{H}$, $\mathrm{CH}_{2}$-Ar, aglycone); 3.66-3.71 (m, $1 \mathrm{H},-\mathrm{OCH}_{2-}$ aglycone+ $1 \mathrm{H}$, sugar); 2.92-3.97 (m, $1 \mathrm{H},-\mathrm{OCH}_{2}-$ aglycone $+1 \mathrm{H}$, sugar); 4.03-4.06 (m, 1H, sugar); 4.15-4.20 (m, 2H, sugar); 4.72-4.92 (m, 6H, sugar); 5.07-5.12 (m, 1H, sugar); 6.68 (d, $1 \mathrm{H}, J=16.4 \mathrm{~Hz}$; $\left.\mathrm{CH}=\mathrm{CH}_{\mathrm{a}}-\mathrm{COO}-\right)$; 7.10-7.16 (m, 3H, aromatic); $7.33\left(\mathrm{~d}, 1 \mathrm{H}\right.$, aromatic), 7.70-7.74 (m, 3H; 2H aromatic+ $\left.1 \mathrm{H} \mathrm{Ar}-\mathrm{CH}_{\mathrm{b}}=\mathrm{CH}\right)$; ESI MS: $m / z$ 1130.1 Da $[\mathrm{M}+\mathrm{H}]^{+}, \mathrm{C}_{56} \mathrm{H}_{72} \mathrm{O}_{24}$ Mol. Wt. 1129.16. 


\subsubsection{Synthesis of Verbascoside Pentapropionate (VPP)}

$\mathrm{Et}_{3} \mathrm{~N}(2.5 \mathrm{~mL})$ was added to a solution of ES1 $(518 \mathrm{mg}, 0.46 \mathrm{mmol})$ in anhydrous $\mathrm{MeOH}(6 \mathrm{~mL})$. The mixture was stirred at room temperature under argon atmosphere for $5 \mathrm{~h}$ and then neutralized by adding of formic acid $(3 \mathrm{~mL})$ at $0{ }^{\circ} \mathrm{C}$. Next the mixture was evaporated under reduced pressure. The crude residue was dissolved in AcOEt and wash with Brine. The organic phase was than dried $\left(\mathrm{Na}_{2} \mathrm{SO}_{4}\right)$, filtered and the solvent evaporated under reduced pressure. The residue obtained was purified by silica gel column chromatography (eluent: $\mathrm{CH}_{2} \mathrm{Cl}_{2} / \mathrm{MeOH}, 98 / 2$, v/v), gave VPP (36\% yield) as a white foam. ${ }^{1} \mathrm{H}-\mathrm{NMR}\left(400 \mathrm{MHz}, \mathrm{DMSO}-d_{6}\right): \delta(\mathrm{ppm}) 0.79\left(\mathrm{t}, 3 \mathrm{H}, \mathrm{CH}_{3}\right) ; 0.92-1.02(\mathrm{~m}$, $15 \mathrm{H}, 5 \mathrm{x} \mathrm{CH}$ ); 1.9-2.05, 2.12-2.34, (m, 10H sugar); 2.58-2.62 (m, 2H, $\left.\mathrm{CH}_{2}-\mathrm{Ar}\right) ; 3.50-3.52$ (m, 1H, $-\mathrm{OCH}_{2}$ - aglycone); 3.65-3.75 (m, 1H, sugar); 3.85-3.9 (m, 1H, - $\mathrm{OCH}_{2}$ - aglycone $+1 \mathrm{H}$ sugar); $4.0(\mathrm{~d}$, $1 \mathrm{H}$, sugar); 4.14-4.2 (m, 2H, sugar); 4.65 (d, 1H, sugar); 4.76-4.88 (m, 5H, sugar ); $5.03(\mathrm{t}, 1 \mathrm{H}$, sugar); 6.21(d, $\left.1 \mathrm{H}, J=16, \mathrm{CH}=\mathrm{CH}_{\mathrm{a}}-\mathrm{COO}\right)$ ); 6.4 (dd, 1H, aromatic); 6.56 (d, 1H, aromatic); 6.60 $(\mathrm{d}, 1 \mathrm{H}$, aromatic); $6.73(\mathrm{~d}, 1 \mathrm{H}$, aromatic); $7(\mathrm{~d}, 1 \mathrm{H}$, aromatic); $7.03(\mathrm{~s}, 1 \mathrm{H}$, aromatic); $7.51(\mathrm{~d}, 1 \mathrm{H}$, $\left.J=16 \mathrm{~Hz}, \mathrm{Ar}-\mathrm{CH}_{\mathrm{b}}=\mathrm{CH}\right) ; 8.65(\mathrm{~s}$, broad , 2H, 2x, OH); 9.2 (s, broad, 1H, OH); 9.65 (s, broad, 1H, $\mathrm{OH})$; ESI MS: $m / z$ 891.6 Da [M+H] ${ }^{+}, \mathrm{C}_{43} \mathrm{H}_{54} \mathrm{O}_{20} \mathrm{Mol}$. Wt. 890.88 .

\subsection{Antioxidant Activity Assays}

\subsubsection{Photochemiluminescence (PCL) Method}

The PCL assay, based on the methodology of Popov and Lewin [14], was used to measure the antioxidant activity of extracts with a Photochem ${ }^{\circledR}$ apparatus (Analytik Jena, Leipzig, Germany) against superoxide anion radicals generated from luminol, a photo-sensitizer, when exposed to UV light (Double Bore ${ }^{\circledR}$ phosphorus lamp, output $351 \mathrm{~nm}, 3 \mathrm{mWatt} / \mathrm{cm}^{2}$ ). The antioxidant activity was measured using both ACW (Antioxidant Capacity of Water-soluble substance) and ACL (Antioxidant Capacity of Liposoluble substance) kits provided by the manufacturer, designed to measure the antioxidant activity of hydrophilic and lipophilic compounds, respectively [15]. For ACW studies, the luminol reagent and Trolox work solution were freshly prepared according to the ACW protocol. The presence of Trolox (or any other antioxidants from the extracts) retarded luminescence for a period: hence, a lag time was noted before a signal was measured. The duration of the lag, which is calculated by the computer software from the first derivative of the detector signal at its turning point and intersection with the x-axis, was plotted against the concentration of Trolox added to the assay medium. The concentration of the added extract solution was such that the generated luminescence fell within the limits of the standard curve. Therefore, the lag time (seconds) for the ACW assay was used as the radical scavenging activity and the antioxidant capacity calculated by comparison with a Trolox standard curve and then expressed as micromoles of Trolox per gram of dry matter of red fibre. In ACL studies, the kinetic light emission curve, which exhibits no lag phase, was monitored for $180 \mathrm{~s}$ and expressed as micromoles of Trolox per gram of dry matter. The areas under the curves were calculated using the PCLsoft control and analysis software. As greater concentrations of Trolox working solutions were added to the assay medium, a marked reduction in the magnitude of the PCL signal and hence the area calculated from the integral was observed. This inhibition was used as a parameter for quantification and related to the decrease in the integral of PCL intensities caused by 
varying concentrations of Trolox. The observed inhibition of the signal was plotted against the concentration of Trolox added to the assay medium. The concentration of the added extract solution was such that the generated luminescence during the $180 \mathrm{~s}$ sampling interval fell within the limits of the standard curve. The extracts for ACW and ACL measurements were centrifuged (5 min at 16,000 g) prior to analysis. The antioxidant assay was carried out in triplicate for each sample, and $20 \mu \mathrm{L}$ of the diluted extract $(1: 40, \mathrm{v} / \mathrm{v})$ in HPLC-grade water (ACW) or HPLC-grade methanol (ACL) was sufficient to correspond to the standard curve.

\subsection{Stability Studies}

\subsubsection{Preparation of Solutions}

The samples were prepared using verbascoside and VPP in different aqueous solutions. Verbascoside was solubilized in $\mathrm{EtOH} / \mathrm{H}_{2} \mathrm{O}$ (80:20) and in buffered aqueous solutions with the following $\mathrm{pH}$ values: 5, 6, 7. VPP was only solubilized in the $\mathrm{EtOH} / \mathrm{H}_{2} \mathrm{O}$ mixture. The samples have been divided in two separate series, called "dark" (naturally preserved in the absence of light.) and "oven" (subjected to accelerated aging in an oven at $40{ }^{\circ} \mathrm{C}$ ).

\subsubsection{Cosmetic Matrices}

In this study, verbascoside and VPP in different formulations have been subjected to accelerated aging in an oven at $40{ }^{\circ} \mathrm{C}$, and analyzed by HPLC with the aim to assess their content over time. The study was carried out on three different formulations, containing $0.3 \%$ of the active ingredient. The formulations tested are as follows:

1. INCI: aqua, glycerin, glyceryl stearate, Ceteareth-20, Ceteareth-12, cetyl palmitate, cetearyl alcohol, dimethicone, caprylic/capric triglyceride, dicapryl carbonate, Symdiol 68T (as preservative agent for VPP formulations) or EUXYLPE9010 (as preservative agent for verbascoside formulations). From a technological point of view, it is an $\mathrm{O} / \mathrm{A}$ cosmetic formulation.

2. INCI: Nomcort T.I.O., Nomcort HK-G, Kester Wax K82D, Covabead LH170, denaturated EtOH (for VPP) or water (for verbascoside). From a technological point of view it's a cosmetic formulation that doesn't contain silicone.

3. INCI: DubPPD1 (hydrogenated palm kernel glycerides, hydrogenated palm glycerides). From a technological point of view it's a pharmaceutical formulation like that used in suppositories.

\subsubsection{HPLC Methods}

The mobile phase consisted of water/acetonitrile (95:5, $0.01 \quad \mathrm{M} \mathrm{H}_{3} \mathrm{PO}_{4}$ ) (solvent $\mathrm{A}$ ) and acetonitrile/water (95:5, 0.01 $\left.\mathrm{M} \mathrm{H}_{3} \mathrm{PO}_{4}\right)$ (solvent B).

(1) Verbascoside: in this study, verbascoside in solution and in formulations has been subjected to accelerated aging in an oven at $40{ }^{\circ} \mathrm{C}$, and then analyzed by HPLC with the aim to assess the content of this active ingredient over time. The determination is carried out under isocratic conditions (solvent A: 83\%/solvent B: 17\%). Separation was monitored with absorbance detection at $330 \pm 8 \mathrm{~nm}$. The flow rate was $1.0 \mathrm{~mL} / \mathrm{min}$, the injection volume was $5 \mu \mathrm{L}$ and all separations were performed at $27^{\circ} \mathrm{C}$. 
(2) VPP: in this study, VPP in solution and in formulations has been subjected to accelerated aging in an oven at $40{ }^{\circ} \mathrm{C}$, and analyzed by HPLC with the aim to assess the content of this active ingredient over time. The determination was carried out under isocratic conditions (solvent A: 45\%/solvent B: $55 \%$ ). Separation was monitored with absorbance detection at $338 \pm 8 \mathrm{~nm}$. The flow rate was $1.2 \mathrm{~mL} / \mathrm{min}$, the injection volume was $5 \mu \mathrm{L}$ and all separations were performed at $27^{\circ} \mathrm{C}$.

\subsubsection{Statistical Evaluations}

Relative standard deviations and statistical significance (Student's t test; $\mathrm{P} \leq 0.05$ ) were given where appropriate for all data collected. One-way ANOVA and LSD post hoc Tukey's honest significant difference test were used for comparing the bioactive effects of different samples. All computations were made using the statistical software STATISTICA 6.0 (StatSoft Italia srl).

\section{Conclusions}

The antioxidant network protects cells against oxidative injury, and when oxidative stress overwhelms this network, the subsequent modification of the cellular redox apparatus leads to an alteration of cell homeostasis leading to degenerative processes. In the dermocosmetic field, the topical application of antioxidants is often suggested as a possible strategy to prevent and modulate oxidative skin damage. We have demonstrated, in the past, that molecular combinations of different antioxidants significantly improve the efficacy of formulations where they are included [22,23]. Recently, we have investigated verbascoside as a natural model of a molecular combination (centaur approach); indeed its structure is a combination of three different moieties: two antioxidants and a sugar part. Continuing our studies on this molecule, we have prepared a possible pro-verbascoside form (VPP) by means of derivatization of the sugar hydroxyl groups but keeping free the active phenol moieties. Different formulations and $\mathrm{pH}$ conditions were investigated for both verbascoside and VPP. The results of the present study shows that the (water free) cosmetic formulation 2 is more stable than formulation 1 and that formulation composition and the $\mathrm{pH}$ values can influence in the stability of verbascoside. Moreover, the stability and antioxidant properties of verbascoside and its derivative VPP obtained by a synthetic modification in order to explore their potential as cosmeceutical and pharmaceutical ingredients should be of great interest in the dermocosmetic and medicinal fields. Our studies demonstrate the potential of this molecule which have been obtained from a sustainable biotechnology platform starting from meristemic cells.

\section{Acknowledgments}

We thank the Ministry of Education and Research (PRIN, Grant 20082L3NFT_003), IRB and Ambrosialab for financial support and Elisa Durini and Alberto Casolari for NMR analysis.

\section{Conflict of Interest}

The authors declare no conflict of interest. 


\section{References}

1. Gutteridge, J.M. Free radicals in disease processes: A compilation of cause and consequence. Free Radic. Res. Commun. 1993, 19, 141-158.

2. Becker, L.B. New concepts in reactive oxygen species and cardiovascular reperfusion physiology. Cardiovasc. Res. 2004, 61, 461-470.

3. Masella, R.; di Benedetto, R.; Vari, R.; Filasi, C.; Giovannini, C. Novel mechanisms of natural antioxidant compounds in biological system: Involvement of glutathione and glutathione related enzymes. J. Nutr. Biochem. 2005, 16, 577-586.

4. Dal Toso, R.; Melandri, F. Ingredienti cosmetici di origine vegetale per via biotecnologica. Cosmet. Technol. 2009, 12, 25-31.

5. Aleo, E.; Ricci, R.; Passi, S.; Cataudella, S. A novel cyt- $\mathrm{H}_{2} \mathrm{O}_{2}$-chemiluminescenes assay for measuring the reducing/antioxidant capacity on hydrophilic and lipophilic antiooxindants and biological samples. Prog. Nutr. 2005, 3, 154-182.

6. Avila Avecedo, J.G.; Castañeda, C.M.; Benitez, F.J.; Durán, D.A.; Barroso, V.R.; Martínez, C.G.; Muñoz, L.J.L.; Martínez, C.A.; Romo de Vivar, A. Photoprotective activity of Buddleja scordioides. Fitoterapia 2005, 76, 301-309.

7. Korkina, L.G.; Mikhalchik, E.; Suprun, M.; Pastore, S.; Dal Toso, R. Molecular mechanisms underlying wound healing and anti-inflammatory properties of naturally occurring biotechnologically produced phenylpropanoid glycosides. Cell. Mol. Biol. 2007, 53, 78-83.

8. Mazzon, E.; Esposito, E.; di Paola, R.; Riccardi, L.; Caminiti, R.; Dal Toso, R.; Pressi, G.; Cuzzocrea, S. Effects of verbascoside biotechnologically produced by Syringa vulgaris plant cell cultures in a rodent model of colitis. Naunyn Schmiedebergs Arch. Pharmacol. 2009, 380, 79-94.

9. Kostyuka, V.A.; Potapovich, A.I.; Suhan, T.O.; de Luca, C.; Korkina, L.G. Antioxidant and signal modulation properties of plant polyphenols in controlling vascular inflammation. Eur. J. Pharmacol. 2011, 658, 248-256.

10. Speranza, L.; Franceschelli, S.; Pesce, M.; Reale, M.; Menghini, L.; Vinciguerra, I.; de Lutiis, M.A.; Felaco, M.; Grilli, A. Antiinflammatory effects in THP-1 cells treated with verbascoside. Phytother. Res. 2010, 24, 1398-1404.

11. Esposito, E.; Mazzon, E.; Paterniti, I.; Dal Toso, R.; Pressi, G.; Caminiti, R.; Cuzzocrea, S. PPAR- $\alpha$ contributes to the anti-Inflammatory activity of verbascoside in a model of inflammatory bowel disease in mice. PPAR Res. 2010, $2010,917312$.

12. Buzzoni, L.; Molesini, S.; Vertuani, S.; Manfredini, S.; Nicotra, G.; Vicentini, S. Ricerca, sviluppo ed attività antiossidante di estratti di Plantago lanceolata titolati in acteoside. Natural 1 2009, 83, 60-67.

13. Lupo, M.P. Antioxidants and vitamins in cosmetics. Clin. Dermatol. 2001, 19, 467-473.

14. Lewin, G.; Popov, I. Oxidants and antioxidants part B-antioxidative homeostasis: Characterization by means of chemiluminescent technique. Methods Enzymol. 1999, 300, 437-456.

15. Popov, I.; Lewin, G. Photochemiluminescent detection of antiradical activity III: A simple assay of ascorbate in blood plasma. J. Biochem. Biophys. Methods 1994, 28, 277-282. 
16. Minghetti, A. Una tecnologia innovativa per la produzione di principi attivi da piante. Colture cellulari di origine vegetale. Natural 1 2003, 20, 54-57.

17. Rice-Evans, C.; Miller, N.J.; Paganga, G. Structure-antioxidant activity relationship of flavonoids and phenolic acids. Free Radic. Biol. Med. 1996, 20, 933-956.

18. Foley, S.; Navaratnam, S.; McGarvey, D.J.; Land, E.J.; Truscott, T.G.; Rice-Evans, C. Singlet oxygen quenching and redox properties of hydroxycinnamic acids. Free Radic. Biol. Med. 1999, 26, 1202-1208.

19. Didry, N.; Seidel, V.; Dubreuil, L.; Tillequin, F.; Bailleul, F. Isolation and antibacterial activity of phenylpropanoid derivatives from Ballota nigra. J. Ethnopharmacol. 1999, 67, 197-202.

20. Xiong, Q.; Tezuka, Y.; Kaneko, T.; Li, H.; Tran, L.Q.; Hase, K.; Namba, T.; Kadota, S. Inhibition of nitric oxide by phenylethanoids in activated macrophages. Eur. J. Pharmacol. 2000, 400, 137-144.

21. Miyazawa, M.; Hisama, M. Antimutagenic activity of phenylpropanoids from clove (Syzygium aromaticum). J. Agric. Food Chem. 2003, 51, 6413-6422.

22. Jaulsin, M.L.; Vertuani, S.; Durini, E.; Buzzoni, L.; Ciliberti, N.; Verdecchia, S.; Palozza, P.; Meier, T.; Manfredini, S. Protective effects of Fe-Aox29, a novel antioxidant derived from a molecular combination of Idebenone and vitamin E, in immortalized fibroblasts and fibroblasts from patients with Friedreich Ataxia. Mol. Cell. Biochem. 2007, 302, 79-85.

23. Napolitano, M.; Avanzi, L.; Manfredini, S.; Bravo, E. Effects of new combinative antioxidant FeAOX-6 and alpha-tocotrienol on macrophage atherogenesis-related functions. Vascul. Pharmacol. 2007, 46, 394-405.

Sample Availability: Samples of the compounds are available from the authors.

(C) 2011 by the authors; licensee MDPI, Basel, Switzerland. This article is an open access article distributed under the terms and conditions of the Creative Commons Attribution license (http://creativecommons.org/licenses/by/3.0/). 